\title{
Erratum to: Laccaria bicolor S238N improves Scots pine mineral nutrition by increasing root nutrient uptake from soil minerals but does not increase mineral weathering
}

\section{Christophe Calvaruso • Marie-Pierre Turpault •}

Stéphane Uroz • Elisabeth Leclerc • Antoine Kies • Pascale Frey-Klett

Published online: 3 August 2011

(C) Springer Science+Business Media B.V. 2011

Erratum to: Plant Soil (2010) 328:145-154

DOI 10.1007/s11104-009-0092-0

Owing to an error during submission, the authors' first and last names were interchanged in the originally published article. The correct names are given here.

Responsible Editor: Katharina Pawlowski.

The online version of the original article can be found at http:// dx.doi.org/10.1007/s11104-009-0092-0.

C. Calvaruso $\cdot$ M.-P. Turpault $(\triangle)$

INRA, UR1138 "Biogéochimie des Ecosystèmes

Forestiers",

Centre de Nancy,

54280 Champenoux, France

e-mail: turpault@nancy.inra.fr

C. Calvaruso

e-mail: chriscalva@hotmail.com

S. Uroz $\cdot$ P. Frey-Klett

INRA-UHP, UMR1136 "Interactions Arbres-

Microorganismes",

Centre de Nancy,

54280 Champenoux, France

E. Leclerc

Andra, Direction Scientifique/Service Transferts, 92298 Châtenay-Malabry, France

C. Calvaruso $\cdot$ A. Kies

Université du Luxembourg "Physique des Radiations",

Campus Limpersberg,

1511 Luxembourg, Luxembourg 